\title{
Informed Parsing for Coordination with Combinatory Categorial Grammar
}

\author{
Jong C. Park Hyung Joon Cho \\ park@cs.kaist.ac.kr hjcho@nlp.kaist.ac.kr \\ Computer Science Division and \\ Advanced Information Technology Research Conter \\ Korea Advanced Institute of Science and Technology \\ 373-1 Gusung-dong, Yusong-gu, Dacjon 305-701, KOREA
}

\begin{abstract}
Coordination in natural language hampers efficient parsing, especially due to the multipole and mostly unintended candidate conjuncts/disjuncts in a given sentence that shows structural ambiguity. The problem gets more scrious in a combinatory catcegorial grammar framework, which is woll known for its compctent treatment of coordination, as the flexilility of syntactic analysis often strikes back as sputrious ambiguity. We propose to address these ambiguities with predicate argument structures and scmantic co-occurrence similarity information, and present encouraging results.
\end{abstract}

\section{Introduction}

Sentences with coordination contain multiple phrases of like syntactic typo. When the given sentence shows structural ambiguity, there may be multiple pairs of candidates for: possible conjuncts/disjuncts, usually a single pair of which is identified as intended by human language understanders. Parsing for coordination should thus find the exact syntactic boundaries of these "intended" conjuncts/disjuncts. P'revious work employed a preprocessing module for parsing to work on a constrained range of the candidate conjuncts/disjuncts (Kurohashi \& Nagao, 1994; Yang, 1995; Okumura \& Muraki, 1994).

Combinatory categorial grammars (Stcedman, 1990; 2000) are known to explain a wide range of syntactic phenomena, such as coordination, extraction and long distance dependency, without employing the notions of movement and empty categories. While CCGs offer explanations for various natiural language phenomena with limited combinatory rules such as type raising and function composition, these are also well known to increase the complexity of parsing, giving rise to quite a few irrelevant syn- tactic analyses as well as relevant ones, a phenomenon often called as spurious ambiguity.

In this paper, we propose to address the two types of ambiguity with predicate argument. structures and scmantic co-occurrence similarity information. ${ }^{1}$ For a more concrete discussion, we focus in this paper on coordination in Korean. First, related work is reviewed in $\xi 2$. The two types of ambiguity are then discussed in $\$ 3$. We also examine the characteristics of coordination in a corpus in $\$ 4$. In $\$ 5$, we slow our proposal to enhance parsing efficiency, with encouraging experimental results.

\section{Related Work}

\subsection{Conjunct Identification}

Finst, we review three of the techniques that attempt to narrow down the candidate conjuncts.

\subsubsection{Complex Information}

Approaches that use complex information are based on the assumption that there are various clues for morphological and semantic similarity between the pair of matching conjuncts/disjuncts. The usual measure for such similarity includes the part-of-speech (POS) feature information. The proposal by Agarwal and Boggess (1992) for English coordination utilizes a semi-parser for the assignment of pertinent semantic and morphological information to cach lexical item in a given sentence. The procedure for the conjunct/disjunct identification with this information is summarized below.

1. Keep pushing the lexical items in a given sentence into the stack until a coordination item such as and, or, but, etc, is encountered.

\footnotetext{
${ }^{1}$ This work was supported by the Korea Science and Engineering Foundation (KOSEF) through AITrc. The second author is now with SK Teletech in Korea.
} 
2. With the coordination item, treat the immediately following phrase as the post-conjunct/disjunct.

3. Pop the lexical items from the stack one by one, comparing their morphological and/or semantic features with those of the post-conjunct/disjunct.

The reported precision is $81 \%$, but the method identifies only the starting positions of the conjuncts/disjuncts.

The method proposed by Okumura and $\mathrm{Mu}-$ raki (1994) for English coordination looks into the symmetric patterns of conjuncts/disjuncts. These symmetric patterns are classified into four categorics: phrasal/clausal patterns, lexical patterns, morphological patterns, and complex patterns. The first three patterns are defined in terms of the respective features in lexical items. The best match among all the possible word sequences with these features before and after the coordination item is passed over to the parser. The reported precision is $75 \%$. This method assigns various weights to the features for the measure of the symmetric patterns. It is not clear if the wcight assignment mothod is principled and if it can also address conjuncts/disjuncts with ellipsis.

\subsubsection{Co-Occurrence Information}

Yang (1995) utilized co-occurrence information for the resolution of structural ambiguity in $\mathrm{Ko}_{\mathrm{O}}$ rean noun phrase coordination. The method looks up the related pair of nouns and verbs from a large corpus and uses the statistics on the case information of the nouns with respect to the given verb for the similarity information among nouns. The co-occurrence similarity $\operatorname{DSim}\left(n_{1}, n_{2}\right)$ between the nouns $n_{1}$ and $n_{2}$ is defined as follows, which incorporates the prediction that the similarity of the two nouns is higher when they are more frequently used with the same vorb of the same syntactic category.

$$
D \operatorname{Sim}\left(n_{1}, n_{2}\right) \equiv \frac{2 \cdot \Sigma_{g \in G}\left|C V_{g}\left(n_{1}, n_{2}\right)\right|}{\Sigma_{g \in G}\left|V_{g}\left(n_{1}\right)\right|+\Sigma_{g \in G}\left|V_{g}\left(n_{2}\right)\right|}
$$

where ${ }^{2}$

- $G \equiv\{s u b j, o b j, l o c a$, inst, modi $\}$

- $V_{g}(n) \equiv\left\{v \mid v\right.$ is a verb such that $\left.f_{g}(n, v) \geq 1\right\}$

- $\left|V_{g}(n)\right| \equiv \sum_{v \in V_{g}(n)} f_{g}(n, v)$

${ }^{2} f_{g}(n, v)$ is the number of times that noun $n$ of type $g$ occurs with verb $v$ in the same sentence in a corpus.

$$
\begin{aligned}
& \text { - } C V_{g}\left(n_{1}, n_{2}\right) \equiv V_{g}\left(n_{1}\right) \cap V_{g}\left(n_{2}\right) \\
& \text { - }\left|C V_{g}\left(n_{1}, n_{2}\right)\right| \equiv \sum_{v \in C V_{g}\left(n_{1}, n_{2}\right)} \min \left\{f_{g}\left(n_{1}, v\right), f_{g}\left(n_{2}, v\right)\right\}
\end{aligned}
$$

This method makes it possible to resolve the syntactic ambiguity due to coordination in Korean as illustrated in (1) and (2). ${ }^{3}$ DSim is used to predict correctly that 'suthayk' (stack) is in coordination with ' $k$ hyu' (queue) and not with 'ycy' (example). Likewise, 'kyesan' (computation) and 'thamsayk' (search) are correctly identified to be in coordination with each other.

(1) suthayk- kwa khyu-uy ycy

stack-co queue-poss example

example(s) of stack(s) and queuc(s)

$D S i m$ (suthayk,khyu) $=0.319$

$D \operatorname{Sim}($ suthayk,ycy) $=0.053$

(2) haysing hamswu-uy kycysan-kwa pekheys-uy thamsayk-cy soyotoynun sikan

hashing function-poss computation-co bucket-poss search-loc spending time

the time spent for the computation of the hashing function and the search for the bucket

$D \operatorname{Sim}($ kycysan, pekhcys $)=0.024$

$D \operatorname{Sim}($ kycysan, thamsayk) $=0.303$

$D \operatorname{Sim}($ kycysan, sikan $)=0.067$

In addition to the fact that the method may suffer from data sparseness, it is also not directly applicable when nouns are polysemous or when the conjunct/disjunct nouns have weak semantic similarity (cf. (3)).

(3) i notu-nun teyitha yoso- wa lisutu-uy taum wensolul cisiha-nun phointhe-lul phohamha-n-ta

this node-nom data element-co list-poss next element-acc indicate-un pointer-acc contain-presdecl

"this node contains the data clement and the pointer that indicates the next element in the list'

Its reported precision is $85.8 \%$, and the recall $95.9 \%$. We note that this co-occurrence similarity information is useful when large-scale linguistic knowledge bases such as WordNet are not available for the language in question.

\subsubsection{Part-Of-Speech Patterns}

The method with Pos patterns (Park, 1998) extracts sentences with coordination from a pos-tagged corpus, trains the system with the POS patterns of the left- and rightconjuncts/disjuncts, and resolves the ambiguity with trained Pos patterns (cf. (4)).

\footnotetext{
${ }^{3}$ Coordination items are shown in a box. We use the Yale notation for transcribing Korean alphabets.
} 
(4) na-nun [komjeng ppang- kwa huyn ppang]-ul mokun salam-ul poass-ta

I-nom [black bread-co whitc bread]-acc eat-un person-acc sec-past-decl

'I saw the person who ate black bread and white bread'

The longest pattern is selected when there are multiple candidate ros-patterns. The reported precision is $71.6 \%$. When there is structiunal ambiguity as shown in (5) below, however, it, is difficult to identify the right conjunct if the system considers only POS information, where the longest match is 'mangko-lul swuipha-num nala' (the nation that imports mangos), not the, intended 'mangko' (mango).

(5) phainayphul- ina mangko-lul swuipha-nun nala pincapple-co mango-acc import-un nation

'the nation that imports pincapples or mangos'

\subsection{Combinatory Categorial Grammar for Korean}

Examples of coordination in Korean are shown below.

(6) [chelswu-n1m1 uysa-ka], [yenghi-num sensayngnim-i] toy-ess-ta

[chelswu-top doctor-comp], [ycnghi-top teach-comp] become-past-decl

'Chelswu became a doctor, and Yenghi a teacher"

(7) [kochwukapsi olu-myen kochwu-lul]], [twaycikokikapsi olu-myen twaycikoki-lul] swuipha-n-ta. [pepper-price-nom rise-if pepper-ace], [pork-priconom rise-if pork-acc] import-pres-decl

'(The government) imports peppers if the price for peppers rises, and pork if the price for pork rises'

In CCGs, type raising and function composition rules are typically utilized to come up with single categories for those fragments above in square brackets, often called 'non-standard' constituents. Table 1 shows the reduction rules proposed for Korean (Cho, 2000; Cho and Park, 2000). Figure 1 shows a sample syntactic and semantic derivation of part of (6). Spacc precludes further explanation of the formalism.

\section{Two Types of Ambiguity}

\subsection{Spurious Ambiguity}

CCGs provide two syntactic derivations for the semantically unambiguous sentence (8), as shown in (9) and (10).

(8) chelswu-ka sakwa-lul mek-ess-ta Chelswu-nom apple-acc eat-past-decl
1. [[chelswu-num sakwa-lul] $\mathrm{mek}]-\mathrm{ko}$ [[yenghi-nun ttalki-lul] mek]-nunta

2. [[chelswu-num sakwa-lul] mek]-ko [yenghi-nun [ttalki-lul mek]]-1nunta

3. [chclswu-mun [sakwa-lul mek]]-ko [yenghi-nun ttalki-lul] mek]-nunta

4. [chelswu-nun [sakwa-lul mek]]-ko [yenghi-nun [ttalki-lul mek]]-nunta

Table 2: Example Spurious Ambiguity

'Chelswu ate an apple/apples'

(9) [[chelswu-ka sakwa-lul] mek-ess-ta]

(10) [chelswu-ka [sakwa-lul mok-css-tal]

These distinct syntactic derivations make it possible for a CCG to correctly analyze sentences with coordination, as shown in (11) and (12).

(11) [[chelswu-ka sakwa-lul]], [yenghi-ka titalki-lul]] inck-ess-1al

'Chelswu ate apples and Yenghi strawberries'

(12) [chelswu-ka [[son-ul ssis]- ko [sakwa-lul mok-ess]tal]

'Chelswu washed his hands and ate apples'

Spurious ambiguity refers to the phenomenom of this kind in which there are multiple syntactic derivations, as in (9) and (10), for a fragment; that is semantically umambiguous. ${ }^{4}$ While descriptively justifiable, it nevertheless results in a repcated computation of the same fragments that are semantically indistinct, adversely affecting parsing efficiency. This problem gets more serious with coordination (cf. (13)).

(13) cholswu-nun sakwa-lul mok- ko yenghi-nun ttalkilul mok-nun-ta

'Chelswu cats apples and Yenghi strawberries'

Table 2 shows four syntactic derivations for (13), all with identical semantics.

\subsection{Structural Ambiguity}

The spurious ambiguity as discussed above does not give rise to wrong syntactic analyses, but the structural ambiguity may, as shown in (14).

(14) cengchi-uy canglay-na nongmintul-uy saynghwalsang-ny mwuncay-wa-nun keli-ka mel-ta

\footnotetext{
${ }^{4}$ Note that there are arguments that some of these syntactic derivations are associated with distinct pragmatic functions, so that the ambiguity might not be entircly 'spurious' (Prevost, 1995).
} 


\begin{tabular}{|c|c|c|}
\hline Reduction Rule & Rule Name & Rule Symbol \\
\hline \hline$X / Y Y \rightarrow X$ & Forward Application & $>$ \\
\hline$Y X \backslash Y \rightarrow X$ & Backward Application & $<$ \\
\hline$X \operatorname{conj} X \rightarrow X$ & Coordination & $<\phi^{n}>$ \\
\hline$X / Y Y / Z \rightarrow X / Z$ & Forward Composition & $>B$ \\
\hline$Y \backslash Z X \backslash Y \rightarrow X \backslash Z$ & Backward Composition & $<B$ \\
\hline$X / Y Y \backslash Z \rightarrow X \backslash Z$ & Forward Crossed Composition & $>B_{x}$ \\
\hline$X \rightarrow T /(T \backslash X)$ & Forward Type Raising & $>T$ \\
\hline$X \rightarrow T \backslash(T / X)$ & Backward Type Raising & $<T$ \\
\hline
\end{tabular}

Table 1: Reduction Rules in a CCG for Korean

\begin{tabular}{|c|c|c|c|}
\hline chelswu - nun & uysa - ka & g & sensayngnim - i \\
\hline $\begin{array}{l}\overline{S_{u} /\left(S_{u} \backslash N P_{n}\right)} \\
\lambda f . f \text { chelswu }\end{array}$ & $\begin{array}{c}\left(\overline{\left.S_{u} \backslash N P_{n}\right) /\left(S_{u} \backslash N P_{n} \backslash N P_{c}\right)}\right. \\
: \lambda f, f \text { doctor }\end{array}$ & $\begin{array}{c}\operatorname{conj} \\
\operatorname{and}(X, Y)\end{array}$ & $\begin{array}{cc}\overline{S_{u} /\left(S_{u} \backslash N P_{n)}\right)} & \left(\overline{\left.S_{u} \backslash N P_{n}\right) /\left(S_{u} \backslash N P_{n} \backslash N P_{c}\right)}\right. \\
: \lambda f . f \text { yenghit } & : \lambda f . f \text { teacher }\end{array}$ \\
\hline $\begin{array}{l}S_{u} / \\
: \lambda f .\end{array}$ & $\begin{array}{l}\left(S_{u} \backslash N P_{n} \backslash N P_{c}\right) \\
\text { f doctor }{ }^{\prime} \text { chelswu }\end{array}$ & & $\begin{aligned} & S_{u} /\left(S_{u} \backslash N P_{u} \backslash N P_{c}\right) \\
: \text { iff.f teacher yenghi } & \end{aligned}$ \\
\hline
\end{tabular}

Figure 1: Sample CCG Derivation

politics-poss future-co farmers-poss life-poss problem-wa-top distance-nom far-decl

'(It is) far from the problems of the future of politics or the lives of farmers'

Table 3 shows six of the syntactic derivations. While the semantics makes it clear that only the derivation 2 is the intended one, it is impossible for a parser with only syntactic information to tell the difference. Notice that this problem is not unique to a CCG-bascd parser. While the general solution would obviously require not only semantic information but also pragmatic and discourse information, we examine approaches that take into account only semantic information in this paper.

\section{Coordination in Corpus}

In an attempt to assess the coordination phenomenon in a realistic manner, we examine a pos-tagged corpus available at KAIST. The corpus contains newspaper articles $(40,428$ eojeol), essays $(41,666$ eojeol), textbooks $(50,208$ eojeol), technical documents (2,729 eojeol), novels $(40,498$ eojeol), with the total of 175,524 eojeol and 17,123 sentences. ${ }^{5}$ Table 4 shows the types

\footnotetext{
${ }^{5}$ Eojeol is a unit in Korean that roughly corresponds to space-delimited words in English. Each cojcol contains both the stem and its morphological endings.
}

1. [cengchi-uy canglay]-na [nongmintul]-uy saynghwalsang-uy mwuncey

2. [cengchi-uy canglay]-na [nongmintul-uy saynghwalsang]-11y mwuncey

3. [cengchi-uy canglay]-na [nongmintul-uy saynghwalsang-uy mwuncey]

4. congchi-uy [canglay]-na [nongmintul]-ny saynghwalsang-uy mwuncey

5. congchi-uy [canglay]-na [nongmintul-uy saynghwalsang]-uy mwuncey

6. cengchi-uy [canglay]-na [nongmintul-uy saynghwalsang-11y mwuncey]

Table 3: Example Structural Ambiguity

and frequencies of sentences containing coordination in this corpus. We used PERL scripts to narrow down the sentences meeting certain basic conditions for coordination and manually identified sentences with coordination among those chosen. ${ }^{6}$ Table 4 indicates that coordination is used quite often in Korean.

\section{Parsing for Coordination}

In this section, we present techniques of dealing with coordination for efficient parsing.

\footnotetext{
${ }^{6} \mathrm{~A}$ pos-tagged corpus does not give sufficient information for the fully automatic identification of sentences with coordination, since we also need to take the sentential semantics into account.
} 


\begin{tabular}{|}
\begin{tabular}{|c||c|c|c|c|c||c|}
\hline Coordination ltem & Articles & Essays & Textbooks & Documents & Novels & Total \\
\hline ending & $728(21.4 \%)$ & $1092(33.7 \%)$ & $1101(20.1 \%)$ & $46(40 \%)$ & $2174(44.5 \%)$ & $5142(30.0 \%)$ \\
\hline postposition & $671(19.7 \%)$ & $352(10.7 \%)$ & $812(14.8 \%)$ & $23(20 \%)$ & $320(6.6 \%)$ & $2178(12.7 \%)$ \\
\hline adverb & $91(2.7 \%)$ & $22(0.68 \%)$ & $58(1.1 \%)$ & $6(5.2 \%)$ & $17(0.35 \%)$ & $194(1 \%)$ \\
\hline comma & $154(4.5 \%)$ & $126(3.9 \%)$ & $216(3.9 \%)$ & $21(18.3 \%)$ & $43(0.88 \%)$ & $605(3.5 \%)$ \\
\hline Total Sentences & 3403 & 3239 & 5485 & 115 & 4881 & $17123(100 \%)$ \\
\hline
\end{tabular}
\end{tabular}

Table 4: Characteristics of Coordination in Korean

\subsection{Predicate Argument Structure}

We used the CKY algorithm to implement a CCG parser. As discussed, the presence of spurious ambiguity in a given sentence forces repeated syntactic analyses for fragments with identical semantics. This can be avoided if we use the same cell to record the syntactic analyses with the same semantics. ${ }^{7}$ CCG makes this possible, as both the syntactic and semantic derivations are constructed in tandem. Table 5 shows part of the parsing table for (13). The following shows the relevant syntactic categories.

- chelswu-num, yenghi-num : $s /\left(s \backslash n p_{n}\right)$

- sakwa-lul, ttalki-lul : $\left(s / n p_{n}\right) /\left(s \backslash n p_{n} \backslash n p_{a}\right)$

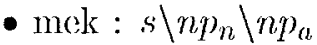

- ko : conj

The colls (C1,R3) and (C5,R3) each contain two syntactic analyses with the same semantics, resulting in four syntactic analyses with the same semantics in the cell $(C 1, R 7)$. Wo can prevent such multiple analyses by not writing into the cell a syntactic analysis with the same recorded scmantics. We thus have one syntactic analysis for each of the cells (C1,R3), (C5,R3) and $(\mathrm{C} 1, \mathrm{R}, 7)$. For longer sentences, we expect a significant reduction in the number of derived syntactic analyses, as also partly verified by our experiments.

\subsection{Co-Occurrence Similarity}

\subsubsection{The Algorithm}

Among the pairs of head nouns of candidate conjuncts/disjuncts, about $88.1 \%$ pairs are identified as semantically related in our corpus. It is thus reasonable to consider only those candidates with some semantic relation. We use the following modification of Yang's (1995) original proposal.

\footnotetext{
${ }^{7}$ Cf. Karttunen, 1989; Eisner, 1996; Komagata, 1999
}

1. Whenever the coordination reduction rule is invoked, check the syntactic categories of the candidate conjuncts.

2 If they are nouns or noun phrases, skip to the next step. Otherwise write them to a coll.

3 Locate the head nouns in the candidate conjuncts.

4 Compute the co-occurrence similarity of the head nouns.

5 If the coordination reduction rule has already been applied to the head noun of the left candidate conjunct, compare the co-occurrence similarity of the recorded and the new. If the co-occurrence similarity of the newly identified candidate conjuncts is stronger, write them to a cell, and delete the existing candidates in the cell. Otherwise, discard the new and retain the old.

6 Update the list of conjuncts whenever there is a newly recorded candidate conjunct.

For the co-occurrence similarity information between nouns, we used another KAIST. corpus that is manually trec-lagged (Lee, 1998). It contains about 31,000 sentences with 352,730 eojeol. The average sentence length is 11.35 words. The domains include newspaper editorials, economy, religion, science fiction, expedition, novels, and history. We have considered only nominative, accusative, adverbial, complemont, and adnominal cases in relation to the verbs for the extraction of co-occurrence similarity information, which is then recorded into a dictionary and is looked up by the parser when it deals with coordination.

\subsubsection{Thesaurus}

The use of co-occurrence similarity information as in (Yang, 1995) suffers from data sparseness, especially since we have a relatively small corpus with fairly unrestricted domains. ${ }^{8}$ For instance, (15) and (16) below show examples of wrong co-occurrence similarity information.

(15) kimchi-wa pap (0)-man cwu-nun kes(0.002252)-ita kimchec-co steamed rice-only give-top thing-clecl.

'(They) served only kimchee and steamed rice' kimchi: 3 vorbs, pap: 9 verbs, kes: 2083 verbs

\footnotetext{
${ }^{8}$ In contrast, Yang used a corpus with one million cojeol and restricted to a computer science domain.
} 


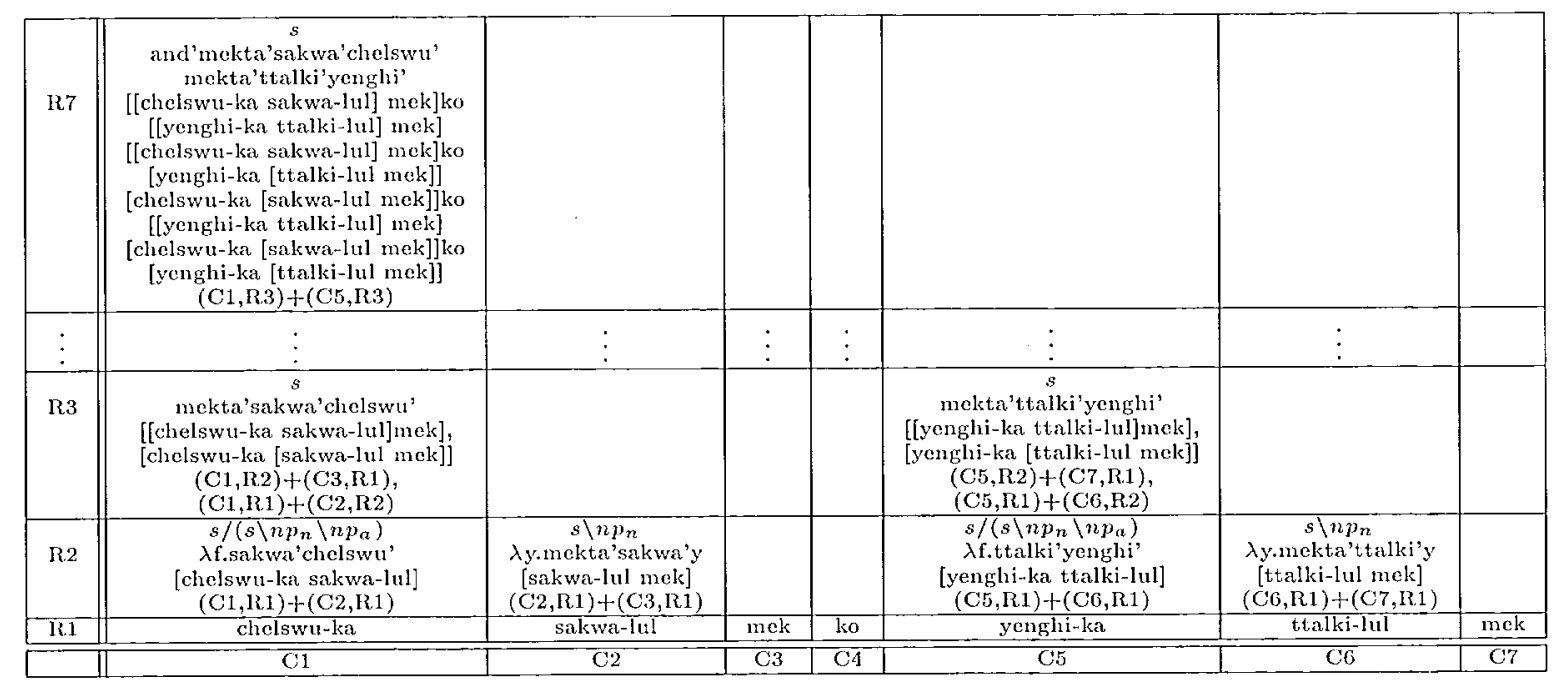

Table 5: Sample CKY Parsing Table

(16) os-kwa cangsingkwu(0)-lul yenkwuha-nun kes $(0.008647)$-iess-supnita.

os: 46 verbs, cangsinkwu: 2 verbs, kes: 2083 verbs

In (15), there are three verbs that occur with 'kimchi' (kimchee), and nine verbs that occur with 'pap' (steamed rice), significantly fewer than those verbs that occur with 'kes' (thing). And in (16), the number of verbs that occur with 'cangsinkwu' (accessory) is smaller than that of the verbs that occur with other nouns. Both result in a wrong analysis. We can use a thesaurus to address this problem.

In a thesaurus, words in the same class are assumed to have related meanings. We can use these class-mate words to compensate for data sparsoness. In constructing a lexicon, we consult the thesaurus when the number of verbs that occur with a given noun falls below a threshold, and let the noun share the data with those in the same class. The thesaurus has the 'word-meaning code' format. The present thesaurus contains slightly more than 1000 nouns that are manually constructed. The classification follows the NTT hierarchy. We have assigned meaning codes to only the most frequently used meanings for polysemous entries. The depth of the hierarchy is 6 . The following shows adjusted results with our thesarus.

(17) kimchi-wa pap $(0.768942)$-man cwu-nun $\underline{\operatorname{kes}(0.008380) \text {-ita. }}$

kimchi: 62 verbs, pap: 64 verbs, kes: 2083 verbs
(18) os-kwa cangsinkwu(0.648276)-lul yenkwuha-nun kes $(0.008647)$ iess-supnita.

os: 46 verbs, cangsinkwu: 69 verbs, kes: 2083 verbs

Table 6 shows the comparison of the methods with various co-occurrence similarity dictionaries using 84 sentences containing noun phrase coordination and structural ambiguity. ${ }^{9}$ It shows that a thesaurus is indeed useful in dealing with data sparseness. In this experi-

\begin{tabular}{|c||c|c|}
\hline & $\mathrm{M} 1$ & $\overline{\mathrm{M}} 2$ \\
\hline \hline Precision & $84.1 \%$ & $88.5 \%$ \\
\hline Recall & $95.3 \%$ & $92.7 \%$ \\
\hline
\end{tabular}

Table 6: Comparison of Different Methods

ment, we have shared the nouns that are associated with fewer than 20 verbs. We have also set the maximum shared examples to 70 and tuned the figures for maximum precision.

\section{$5.3 \quad$ Results}

For the performance evaluation, we have compared three kinds of parsers. A employs only the CKY algorithm. B has the additional module for spurious ambiguity. C utilizes the aforementioned dictionary, in addition to the module for spurious ambiguity. We found out that the

\footnotetext{
${ }^{9} \mathrm{M} 1$ utilizes only the similariy dictionary as defined by Yang (1995). M2 has the extra information from KAIST tree-tagged corpus and the thesaurus.
} 
primary factors for the extra parsing complexity include the number of right conjunct candidates, the presence of modifiers in the left conjunct, and the type of sentences (simple or complex) with noun phrase coordination.

In order to check for the influence of structural ambiguity on parsing efliciency, wo have classified 53 sentences into 4 types, considering modifiers in the left conjunct (cf. Table 7). ${ }^{10}$ Table 8 shows the number of semantic struc-

\begin{tabular}{|c||c|c|}
\hline Sentences & Modifiers (range) & \#t of candidates \\
\hline \hline Type 1 & no & $\leq 3$ \\
\hline Type 2 & yes (unambiguous) & $\geq 4$ \\
\hline Type 3 & yes (ambiguous) & $\leq 3$ \\
\hline Type 4 & yes (ambiguous) & $\geq 4$ \\
\hline
\end{tabular}

\section{Table 7: 'Types of Sentences}

tures as derived by each parser. ${ }^{11}$ The average numbers of semantic: structures derived by $B$ and $C$ are 26.2 and 7.3 , respectively, resulting in the reduction of $72.1 \%$. Table 9 shows

\begin{tabular}{|c||c|c|c|}
\hline & $A$ & 13 & $C$ \\
\hline Type 1 & 189.8 & $\overline{4.3}$ & 2.0 \\
\hline Type 2 & 963 & 32.3 & 12.3 \\
\hline Type 3 & 245.1 & 13.4 & 5.4 \\
\hline Type 4 & - & 83.3 & 16.4 \\
\hline
\end{tabular}

Table 8: Derived Semantic: Structures

the parsing time for each method. ${ }^{12}$ 'The reason that 33 appears gencrally faster than C (except for type 4) is that ("spends extra time on consulting the dictionary database for the sinnilarity. But the average analysis time by $B$ is 298.79 ms, whereas C takes 228.92 ms, making parsing more eflicient in time reduction of $23.39 \%$. Albeit premature, we believe that these results are encouraging.

\section{Concluding Remarks}

Through experiments, we have confirmed that we can address spurious ambignity in a CCG

\footnotetext{
${ }^{10}$ The average sentence lengths of types 1 through 4 are $12.4,16.7,13.3$, and 20.5 morphemes, respectively.

${ }^{11} \mathrm{~A}$ was not able to produce results at all for any of the sentences of type 4, and failed on half of the sentences of type 2 due to insufficient memory. The figures in the table reflect only the successful ones.

${ }^{12}$ We used the statistics package of SICStus Prolog, under Sun Enterprise 250 with 512M13 RAM. There were $23,10,10$, and 10 sontences of types 1 through 4 , resp).
}

\begin{tabular}{|c|c|c|c|}
\hline & $\mathrm{A}$ & $\mathrm{B}$ & $\mathrm{C}$ \\
\hline Type 1 & 1168 & 228 & 277 \\
\hline Type 2 & 5789 & 1001 & 1377 \\
\hline Type 3 & 1284 & 305 & 335 \\
\hline Type 4 & - & 4504 & 2504 \\
\hline
\end{tabular}

Table 9: Average Parsing Times (in ms)

framowork by incorporating predicate argument: structures into the parsing module, and shown that co-occurronce similarity information angmented with a thesaurus helps the parser to deal with structural ambiguity. We leave open the problem of addressing those conjuncts/disjuncts that are noither semantically related nor anticipated by a corpus.

\section{References}

12. Agarwal and L. Boggess. 1992. A simple but useful approach to conjunct identification. ACL, pp. 15-21.

IJ. J. Cho. 2000. Coordinate Constructions in Kortan and I'arsing Issues in Combinatory Catcgorial Grammar. KAIST: MS thesis.

H. J. Cho and .J. C. P'ark. 20000. Combinatory Categorial Grammar for the Syntactic; Semantic, and Discourse Analyses of Coordination in Korean. Journal of KISS: Softuarc and Applications, 27(4), pp. 448-462.

J. Eisner. 1996. Efficient normal-form parsing for Combinatory Categorial Grammar. ACL. pp. 79-86.

I. Karttunnen. 1989. Radical Lexicalism. In Alternative Conceptions of Phrase Structure. U of Chicago Press.

N. Komagata. 1999. Information Structure in Texts: A Computational Analysis of Contextual Appropriateness in I'nglish and Japanese. U of Pennsylvania Ph] thesis.

S. Kurohashi and M. Naggao. 1994. A syntactic analysis method of long. Japanese sentences based on detection of conjunclive structures. CL, 20(4), pp. 507-534.

K. J. Lee. 1998. Stochastic Syntactic Analysis of Korean with Linguistic Propertices. KAIST PhID thesis.

A. Okumura and K. Muraki. 1994. Symmetric pattern matching analysis for English coordinate structures. ANLP, pp. 41-46.

J. S. Park. 1998. Analysis of Coordinate Constructions in Korean with Part-of-Speceh Patterns. KAIST. MS thesis.

S. Prevost. 1995. A Semantics of Contrast and Information Structure for Specifying Intonation in Spoken Language Gencration. U of Pennsylvania PhD thesis.

M. Stcedman. 1990. Gapping as Constituent Coordination. Itinguistics and Philosophy 13, p1. 207-263.

M. Stedman. 2000. The Syntiactic Process. MIT Press.

J. H. Yaug. 1995. Structural Ambiguity Resolution in Korean Noun Phrase Conjunctions Using Co-occurrence Similarity. Journal of KISS (B), 23(3), pp. 311-321. 